\title{
STUDIES OF THE BROMINE SPECTRUM AND DETERMINATION OF TRANSITION PROBABILITIES FOR Br I AND Br II LINES
}

\author{
T. Wujec, A. Baclawsiri, A. Golly and I. Książer \\ Institute of Physics, Opole University, Oleska 48, 45-052 Opole, Poland
}

(Received December 14, 1998; revised version May 7, 1999)

In a wall-stabilized cascade arc at atmospheric pressure in mixtures of argon and bromine vapors thermal plasmas were generated. Arc currents between 25 and $60 \mathrm{~A}$ were used. Applying a spectrometer of medium dispersion, equipped with an optical multichannel analyzer (CCD detector), the spectra. of neutral and singly ionized bromine in the range between 3300 and $10400 \AA$ were recorded. Line intensity measurements, corrected for self-absorption, at different operating conditions of the arc, were performed. A complete set of Br I transitions (5 lines) originating from a common upper level (branching fractions) was measured. In the case of the Br II spectrum intensities of 14 lines were determined. Both sets of lines ( $\mathrm{Br}$ I and Br II) were normalized to an absolute scale using appropriate lifetime values. The uncertainties of the new determined transition probabilities depend mainly on the uncertainties of the lifetime datia taken from the literature. The results are compared to other experimental data. Discrepancies are found to be outside of uncertainties of the measurements.

PACS numbers: $32.10 .-f, 95.30 . \mathrm{Ky}$

\section{Introduction}

The knowledge of atomic structure constants of bromine is of considerable scientific importance because bromine is known as revealing great affinity in forming chemical compounds and because of the possible use of this element in chemical lasers. The knowledge of transition probabilities of spectral lines of various elements is also indispensable for calculations of radiative transfer through stellar atmospheres and diagnostics of cosmic plasma. The principal problem in determination of transition probabilities of spectral lines is to obtain a homogeneous and stable plasma, from which these spectral lines are emitted. A very good source, widely used for excitation of atoms and molecules in atomic spectroscopy, is the wall-stabilized d.c. cascade arc introduced by Maecker [1] and somewhat modified by Shumaker [2]. As a main plasma component (working gas) argon is mostly used 
with a small admixture of the element under study. The plasma generated in such arc reveals (with some exceptions) cylindrical symmetry, is nearly homogeneous along the arc axis and shows a large negative temperature gradient in radial direction. This gradient causes difficulties when heavy elements (e.g. metals), in form of vapors are introduced into the plasma. Vapors usually condense on the disc walls and thus influence the stability of the discharge. This undesirable effect is minimized by applying arc discs allowing to introduce the vapours diluted in the working gas (Goly and Wujec [3], Baruschka and Schulz-Gulde [4]). In this work we used arc discs with chamnels, which enable the arc to be sealed and allow to vary the length of the plasma column containing the bromine admixture. The main purpose of our work was to create plasmas emitting stable bromine spectra and to obtain transition probabilities for $\mathrm{Br} I$ and $\mathrm{Br}$ II lines in the visible and infrared range of spectrum. The description of the experimental method used in this work and results for some other neutral bromine lines were recently published by Wujec et al. [5]. In the present paper also some cletails about the methods of absorption correction for bromine lines are presented.

\section{Experimental}

In order to excite the spectra of bromine atoms and ions, bromine vapours were introduced into an argon plasma generated in a wall-stabilized arc. In Fig. 1 the scheme of the arc and the setup for introduction of argon and bromine vapours into the arc, are presented. Two argon streams (usually $20 \mathrm{l} / \mathrm{h}$ ) were introduced into the electrode areas in order to protect the copper anode and the tungsten cathode from corrosion caused by bromine. The mixture of gases $\left(\mathrm{Ar}+\mathrm{Br}_{2}\right)$ was introduced symmetrically into the central part of the arc (usually $40 \mathrm{l} / \mathrm{h}$ ). In this way a stable operation of the arc for several hours was ensured. The mixture $\left(\mathrm{Ar}+\mathrm{Br}_{2}\right)$ was produced by passing a part of the argon flow through the liquid

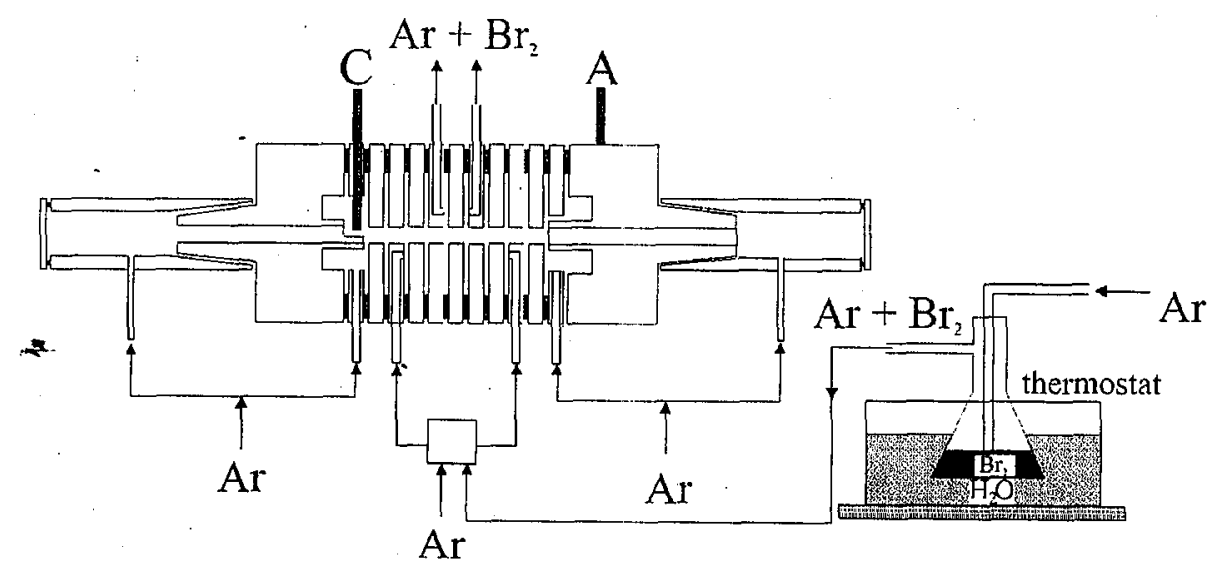

Fig. 1. Scheme of the plasma source used for bromine excitation; $A$ - anode, $C$ cathode. 
bromine kept in a thermostat at $0^{\circ} \mathrm{C}$. In this way it was possible to regulate the amount of bromine introduced into the arc at fixed total amount of the gas mixture. The bromine concentration in the plasma was controlled during the measurements by monitoring the intensities of $\mathrm{Br}$ I lines at $6350 \AA$ or $4477 \AA$. For this purpose the spectrometer MDP-4 was applied.

The radiation of the plasma emitted in end-on direction was recorded applying the spectrograph PGS-2 equipped with an optical multichannel analyzer (OMA-4) controlled by a PC type computer (reciprocal dispersion $0.14 \AA /$ pixel). The plasma column was imaged onto the entrance slit of the spectrometer (magnification 1:1) applying a concave mirror $(f=73 \mathrm{~cm})$. Details of the optical setup are shown schematically in Fig. 2.

Spectra in the wavelength interval $3300 \div 10400 \AA$ were recorded at arc currents between 25 and $60 \mathrm{~A}$. In a single exposition a wavelength interval of $144 \AA$ only could be recorded. The detection of the spectrum, however, was done

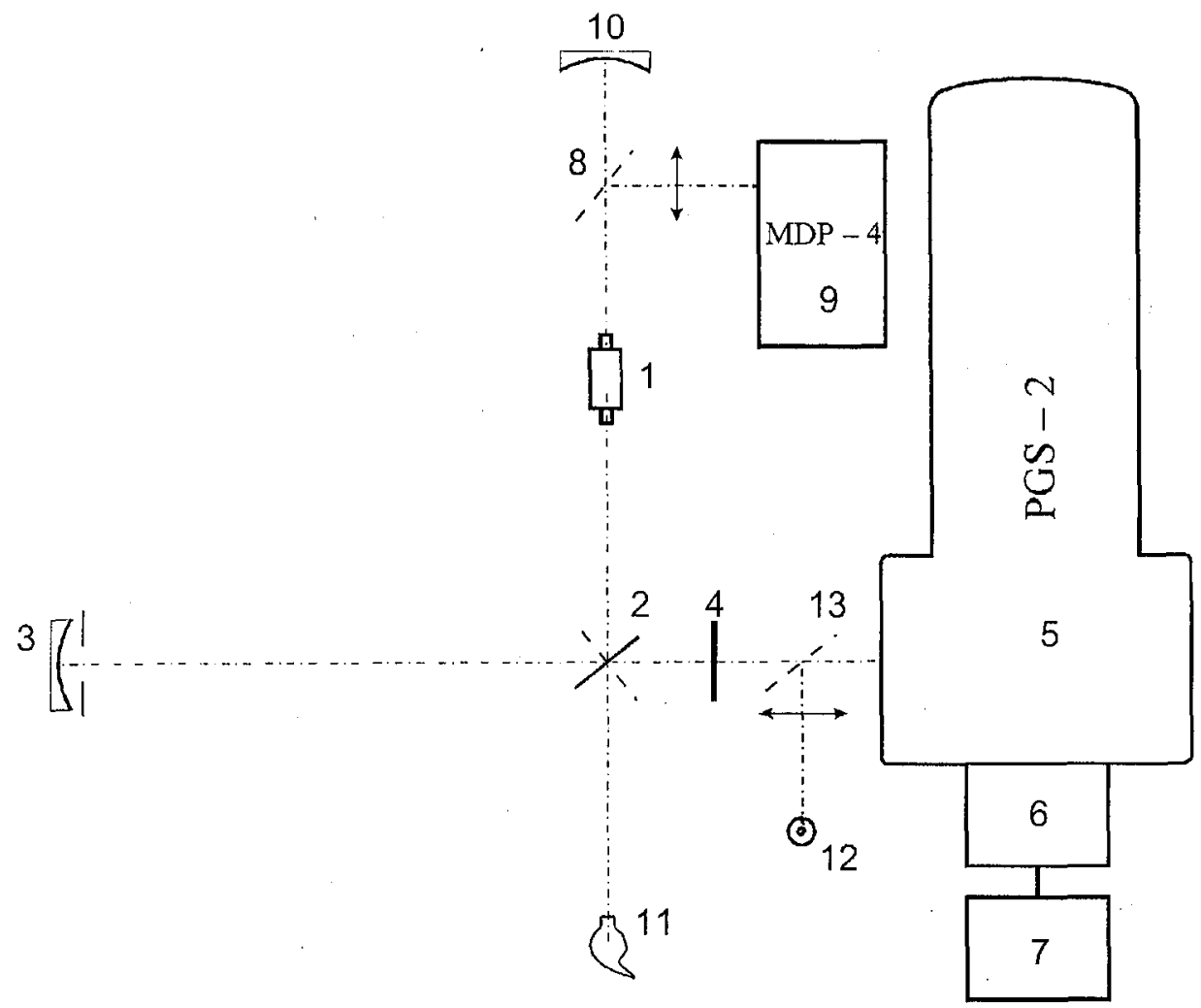

Fig. 2. Schematic diagram of the experimental setup. 1 - wall-stabilized arc, 2 - plane mirror, 3 - concave mirror, 4 - filter, 5 -- grating spectrograph PGS-2, 6 - optical multichannel analyzer OMA-4, 7 - computer (IBM-PC type), 8 - plane mirror, 9 -spectrometer MDP-4, 10 - concave mirror, 11 - tungsten strip lamp, 12 - Plücker tube, 13 - plane mirror. 
in $100 \AA$ steps facilitating the iclentification of the spectrum. The classification of bromine lines and notation of energy levels were taken from Ramanadham and Rao [6], Moore [7] and Tech [8]. The measured spectra were converted to an absolute scale applying a tungsten strip lamp calibrated at National Institute of Standards and Technology. The optical thickness of selected spectral lines was studied in a way outlined below.

\subsection{Self-absorption correction of measured line intensities}

As mentioned above in this experiment the spectrum of the plasma was observed in end-on direction. The total length of plasma layer was about $7 \mathrm{~cm}$. Analyses of preliminary recorded spectra indicate that for some recorded lines it is necessary to consider the radiation transfer along the line of sight and consequently to perform appropriate corrections of the measured line shapes.

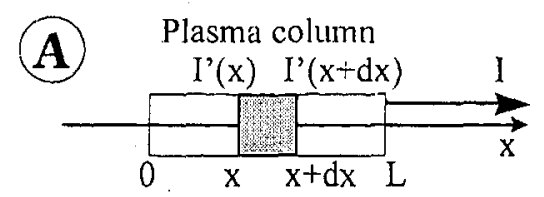

I - observed intensity I' - intensity at the coordinate " $x$ "

B

Mirror Shutter Window Plasma column Window Spectrometer

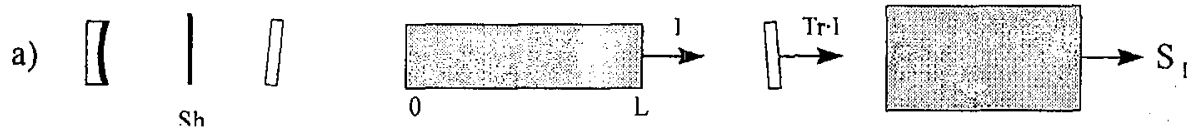

b)

Fig. 3. Scheme of the optical arrangement for measuring of self-absorption. (A) Radiative transfer throngh the plasma column, (B) method of absorption coefficient measurements using the reflected light passing through the plasma.

The radiation transfer through the plasma layer (see Fig. $3 \mathrm{~A}$ ) is given by the equation [9]:

$$
\frac{\mathrm{d} I^{\prime}(\lambda, x)}{\mathrm{d} x}=\varepsilon(\lambda, x)-k(\lambda, x) I^{\prime}(\lambda, x)
$$

where $k(\lambda, x)$ and $\varepsilon(\lambda, x)$ are the local absorption and emission coefficients, respectively. The relationship between quantities $\varepsilon(\lambda)$ and $k(\lambda)$ is given by Kirchhoff's law

$$
\frac{\varepsilon(\lambda)}{k(\lambda)}=P(\lambda, T)
$$

where $P(\lambda, T)$ is the Planck function. 
According to Lochte-Holtgreven [10] a correcting procedure may be performed for lines with optical thickness $k(\lambda) L<0.5$.

Two different correction methods applied in this work are presented below.

\subsubsection{Self-absorption correction based on Firchhoff's law}

Assuming that the radiation originates from an homogeneous plasma layer and taking into account $\mathrm{Eq}$. (2) the solution of Eq. (1) leads to the spectral distribution $\varepsilon(\lambda)$ :

$$
\varepsilon(\lambda)=-\frac{P(\lambda, T)}{L} \ln \left[1-\frac{I^{\prime}(\lambda)}{P\left(\lambda, T^{\prime}\right)}\right],
$$

where $L$ is the length of the plasma and $I^{\prime}(\lambda)$ is the observed spectral intensity distribution. The application of this method requires the knowledge of the plasma temperature.

\subsubsection{Self-absorption correction using the reflected plasma radiation}

The principle of the second method is shown in Fig. 3B. The concave mirror is placed at such position that the plasna is imaged on itself. The spectrum is detected twice: (a) when the shutter $S h$ is closed (signal $S_{1}$ ), and (b) when the shutter is open (signal $S_{2}$ ). The factor $A^{\prime}$ is given by

$$
A^{\prime}=\exp [-k(\lambda) L]
$$

where

$$
k(\lambda)=\frac{1}{L} \ln \left[\frac{S_{2} / S_{1}-1}{R(T r)^{2}}\right],
$$

and $R$ is reffectivity of the mirror and $T r$ is the transmission of the windows. The emission coefficient determined in this way is then given by

$$
\varepsilon(\lambda)=\frac{I^{\prime}(\lambda) k(\lambda)}{1-\exp [-k(\lambda) L]} .
$$

No plasma parameters are required to evaluate the emission coefficient. The instrumental factor $R(T r)^{2}$ may be determined from the signal ratio in the far wings of the line, where the self-absorption is negligible

$$
\frac{S_{2}^{\prime}}{S_{1}^{\prime}}=1+R\left(T r^{2}\right)^{2}
$$

This optical thickness measurementis are very useful in the phase of adjusting the conditions of the experiment. Both described methods of correction of self-absorption lead generally to similar results if the plasma is close to local thermodynamic equilibrium (LTE) conditions. In order to study the self-absorption in bromine lines we performed experiments with the following gas compositions: $20+20,30+10,37+3$, where the first number (in $1 / \mathrm{h}$ ) corresponds to the amount of argon introduced directly to the arc and the second number to the amount of argon flowing through the liquid bromine. For bromine transition probability determination we optimized the concentration of bromine in the plasma in order to be able to correct the measured intensities even for strong lines. After spectral intensity corrections the line shapes were fitted by Lorentz functions and the total 
emission coefficients have been calculated. In this way emission coefficients of 5 $\mathrm{Br} \mathrm{I}$ and $14 \mathrm{Br}$ II lines were determined with a satisfactory accuracy.

The temperature and electron concentration were obtained by assuming LTE conditions and measuring the emission coefficient and the FWHM of the Ar I $4300.1 \AA$ line. The following results were obtained: $T=12000 \mathrm{~K}$ and $n_{e}=$ $(6.3 \pm 0.4) \times 10^{16} \mathrm{~cm}^{-3}$. Both parameters $\left(T, n_{\mathrm{e}}\right)$ are not critical at all for our Br I transition probability determination since the final results are based on branching fractions measurements. In the case of the Br II spectrum the plasma temperature was used for calculations of relative transition probabilities from experimentally derived emission coefficients of the respective lines. At the electron concentration of our argon-bromine plasma $\left(6.3 \times 10^{16} \mathrm{~cm}^{-3}\right)$ the assumption of the Boltzmann distribution among the excited Br II levels is justified.

\section{Determination of transition probabilities}

The most accurate method to determine transition probabilities by the emission method is to measure complete sets of branching ratios originating from a given level $(k)$ with a known lifetime $\tau_{k}$ :

$$
A_{k i}=\frac{1}{\tau_{k}} \frac{I_{k i} \lambda_{k i}}{\sum_{j} I_{k j} \lambda_{k j}}
$$

Transition probabilities $\left(A_{x}\right)$ for other lines originating from different upper levels $(x)$ can be obtained from the formula

$$
A_{x}=A_{0} \frac{I_{x} \lambda_{x} g_{0}}{I_{0} \lambda_{0} g_{x}} \exp \left(\frac{E_{x}-E_{0}}{k T}\right),
$$

where $\lambda_{x}, I_{x}, g_{x}$ and $E_{x}$ are the wavelength, total line intensity, statistical weight and excitation energy of the upper level for the selected line, respectively. The subscript 0 holds for the reference line with known transition probability.

\section{Discussion of results}

In preliminary recorded spectra 155 lines of $\mathrm{Br} I$ were investigated, but many lines were not suitable for intensity measurements with sufficient accuracy. Coincidences with argon lines and strong self-absorption for some bromine lines were observed. Finally only spectra obtained from three different plasma conditions were evaluated for transition probability determination. In the first step transition probabilities for five $\mathrm{Br}$ I lines originating from the upper energy level $\left(5 p^{\prime}{ }^{4} S_{3 / 2}^{\circ}\right)$ were determined using Eq. (8). For normalization the lifetime $\tau=(43 \pm 8) \mathrm{ns}$ was taken from Ref. [11]. The results are listed in Table I. In the last column of Table I the estimated uncertainties are given, which do not exceed $25 \%$. The main contribution to the uncertainties results from the error of the applied lifetime value (19\%). Standard deviations of measured line intensity ratios are very small and amount from less than $1 \%$ to $3 \%$. Generally the agreement of our data with the results of Bengtson et a.l. [12] is not satisfactory. Only in the case of the line at $8131.52 \AA$ the discrepancy does not exceed the uncertainty limit.

For determination of relative intensities of $\mathrm{Br}$ II lines the line a.t wavelength $4816.70 \AA$ was used as a reference standard. The transition probability of this 
TABLE I

Transition probabilities of $\mathrm{Br}$ I lines determined from measurements of branching fractions and applying the lifetime of the level $5 p^{4} S_{3 / 2}^{0}$ for establishing the absolute scale. Our results are compared to those of Bengtson et al. [12].

\begin{tabular}{|c|c|c|c|c|c|}
\hline \multirow{2}{*}{$\begin{array}{c}\text { Wavel. } \\
{[\AA]}\end{array}$} & \multirow{2}{*}{$\begin{array}{c}\text { Lower } \\
\text { level }\end{array}$} & \multirow{2}{*}{$\begin{array}{l}\text { Upper: } \\
\text { level }\end{array}$} & \multicolumn{3}{|c|}{$A_{k i}\left[10^{6} \mathrm{~s}^{-1}\right]$} \\
\hline & & & Ref. [12] & This work & Uncert. \\
\hline 6350.73 & $4 p^{4}\left({ }^{3} P_{2}\right) 5 s^{4} P_{5 / 2}$ & \multirow[t]{5}{*}{$4 p^{4}\left({ }^{3} P_{1}\right) 5 p^{4} S_{3 / 2}^{\circ}$} & & 1.6 & \pm 0.35 \\
\hline 7005.19 & $4 p^{4}\left({ }^{3} P_{2}\right) 5 s^{4} P_{3 / 2}$ & & 12.3 & 3.1 & \pm 0.7 \\
\hline 8131.52 & $4 p^{4}\left({ }^{3} P_{1}\right) 5 s^{4} P_{1 / 2}$ & & 3.8 & 3.6 & \pm 0.8 \\
\hline 8334.70 & $4 p^{4}\left({ }^{3} P_{1}\right) 5 s^{2} P_{3 / 2}$ & & 14.6 & 10.7 & \pm 2.2 \\
\hline 9793.48 & $4 p^{4}\left({ }^{3} P_{0}\right) 5 p^{2} P_{1 / 2}$ & & & 4.3 & \pm 0.9 \\
\hline Lifetime & & $\tau=43 \mathrm{~ns}$ & & $(i)^{-1}=$ & ns \\
\hline
\end{tabular}

TABLE II

Transition probabilities of $\mathrm{Br}$ II lines determined from line intensity measurements. The absolute scale is based on the lifetime value for the level $5 p\left({ }^{4} S\right){ }^{5} P_{1}$ obtained by Martinez et al. [13]. Our results are compared to the only available experimental data of Bengtson et al. [14].

\begin{tabular}{|c|c|c|c|c|c|c|}
\hline \multirow{2}{*}{$\begin{array}{c}\text { Wavel. } \\
{[\AA]}\end{array}$} & \multirow{2}{*}{$\begin{array}{c}E_{k} \\
{\left[\mathrm{~cm}^{-1}\right]}\end{array}$} & \multirow{2}{*}{$\begin{array}{c}\text { Lower } \\
\text { level }\end{array}$} & \multirow{2}{*}{$\begin{array}{l}\text { Upper: } \\
\text { level }\end{array}$} & \multicolumn{3}{|c|}{$A_{k i}\left[10^{8} \mathrm{~s}^{-1}\right]$} \\
\hline & & & & Ref. [14] & This work & Uncert. $^{a}$ \\
\hline 4223.83 & 136607.7 & $5 s^{\prime 1} D_{2}^{\circ}$ & $5 p^{\prime 1} D_{2}$ & & 0.46 & $\mathrm{C}$ \\
\hline 4365.60 & 133601.1 & $5 s^{\prime 3} D_{3}^{\circ}$ & $5 p^{\prime}{ }^{3} P_{2}$ & & 0.44 & $\mathrm{C}$ \\
\hline 4.379 .74 & 151502.3 & $5 p^{\prime 3} D_{1}$ & $5 d^{\prime 3} D_{2}^{\circ}$ & & 3.62 & $\mathrm{D}$ \\
\hline 4622.75 & 131308.7 & $5 s^{\prime 3} D_{2}^{\mathrm{o}}$ & $5 p^{\prime}{ }^{3} F_{3}$ & & 0.14 & C \\
\hline 4678.70 & 131745.7 & $5 s^{\prime}{ }^{3} D_{3}^{\circ}$ & $5 p^{\prime} F_{4}$ & & 0.43 & $\mathrm{D}$ \\
\hline 4704.85 & 115176.2 & $5 s^{5} S_{2}^{\prime}$ & $5 p^{5} P_{3}$ & 1.05 & 0.53 & $\mathrm{C}$ \\
\hline 4719.76 & 130609.9 & $5 s^{\prime 3} D_{1}^{\circ}$ & $5 p^{\prime}{ }^{3} F_{2}$ & & 0.40 & $\mathrm{C}$ \\
\hline 4785.50 & 114818.1 & $5 s^{5} S_{2}^{\circ}$ & $5 p^{5} P_{2}$ & 0.48 & 0.94 & $\mathrm{C}$ \\
\hline 4816.70 & 114682.8 & $5 s^{5} S_{2}^{\circ}$ & $5 p^{5} P_{1}$ & 0.94 & 0.48 & $\mathrm{C}$ \\
\hline 4928.79 & 129965.9 & $5 s^{\prime}{ }^{3} D_{2}^{\circ}$ & $5 p^{\prime}{ }^{3} D_{2}$ & & 0.30 & $\mathrm{D}$ \\
\hline 4930.66 & 130653.9 & $5 s^{\prime}{ }^{3} D_{3}^{\circ}$ & $5 p^{\prime 3} D_{3}$ & & 0.19 & $\mathrm{D}$ \\
\hline 5164.38 & 117834.3 & $5 s^{3} S_{1}^{\circ}$ & $5 p^{3} P_{0}$ & & 0.44 & $\mathrm{D}$ \\
\hline 5182.35 & 117767.6 & $5 s^{3} S_{1}^{\circ}$ & $5 p^{3} P_{2}$ & & 0.35 & $\mathrm{D}$ \\
\hline 5238.23 & 117561.5 & $5 s^{3} S_{1}^{\circ}$ & $5 p^{3} P_{1}$ & & 0.40 & $\mathrm{C}$ \\
\hline
\end{tabular}

${ }^{a} \mathrm{C}-$ uncertainties within $25 \%, \mathrm{D}$ - uncertainties within $50 \%$.

line was determined using the lifetime $\tau=(24 \pm 4)$ ns for the $5 p\left({ }^{4} S\right){ }^{5} P_{1}$ level, measured by Martinez et al. [13]. From this level two transitions to lower levels are possible, resulting in the emission of spectral lines at 4816.7 and $6168.7 \AA$. The contribution to the decay from the intersystem line at $6168.7 \AA$ (lower level 
$\left.4 p^{3}\left({ }^{4} S\right) 5 s^{3} S_{1}\right)$ has not been taken into consideration because at our experimental conditions this line was not observed. Transition probabilities for all other Br II lines were determined applying Eq. (9). In Table II our transition probabilities for $\mathrm{Br}$ II lines are compared to data obtained by Bengtson et al. [14]. The listed data are mean values calculated from 6 independent measurements. Our results in Table II can be clivided into two groups with determination accuracies within $25 \%$ (C) and within $50 \%$ (D). The estimated errors include the determination accuracy of the applied lifetime value $(+19 \%)$.

Our results are about twice smaller than Bengtson's data, however the relative transition probabilities agree well. The discrepancy of the absolute scale results from different normalization procedures applied in our work and by Bengtson and Miller.

\section{Acknowledgments}

This work was supported by the U.S.-Polish Maria Skiłodowska-Curie Joint Fund II, project MEN/NIST-96-260.

\section{References}

[1] H. Maecker, Z. Naturforsch. A 11, 457 (1956).

[2] B. Shumaker, Rev. Sci. Instrum. 32, 65 (1961).

[3] A. Goly, T. Wujec, in: AIP Conf. Proc. 328, Spectral Line Shapes, Vol. 8, Eds. A.D. May, J.R. Drummond, E. Oks, AIP Press, New York 1995, p. 95.

[4] G. Baruschka, E. Schulz-Gulde, Astron. Astrophys. 44, 335 (1975).

[5] T. Wujec, A. Bacławski, A. Golly, I. Ksiązek, J. Quant. Spectrosc. Radiat. Transf. 61, 533 (1999).

[6] R. Ramanadham, K.R.. Rao, Indian J. Phys. 18, 317 (1945).

[7] C.E. Moore, Atomic Energy Levels II. NBS (1972).

[8] J.L. Tech, J. Res. NBS A, Phys. Chem. A 67, 505 (1963).

[9] J. Richter, in: Plasma Diagnostics, Ed. W. Lochte-Holtgreven, North-Holland, Ansterdam 1968, p. 53.

[10] W. Lochte-Holtgreven, in Ref. [9], p. 196.

[11] M.L. Burshtejn, V.A. Komarovskii, Opl. Spektrosk. (USSR) 57, 362 (1984).

[12] D. Bengtson, M.H. Miller, D.W. Koopman, T.D. Wilkerson, Phys. Rev. A 3, 16 (1971).

[13] R. Martinez, I. Merelast, M.N. Sanchez Rayo, F. Castano, J. Phys. B, At. Mol. Opt. Phys. 28, 4563 (1995).

[14] R.D. Bengtson, M.H. Miller, Phys, Rev. A 14, 1915 (1976). 